\title{
Histone deacetylase inhibitor attenuates intestinal mucosal injury in fatally scalded rats
}

\author{
Rui Liu ${ }^{1,2 \#}$, Shu-Ming Wang ${ }^{3 \#}$, Si-Jia Guo ${ }^{2} \wedge$, Ming-Ming $\mathrm{Ma}^{2} \wedge, \mathrm{Yi}-\mathrm{Li} \mathrm{Fu}^{1} \wedge$ \\ ${ }^{1}$ Center for Life Sciences, School of Life Science and Technology, Harbin Institute of Technology, Harbin, China; ${ }^{2}$ Department of Burns, \\ Heilongjiang Provincial Hospital, Harbin, China; ${ }^{3}$ Department of Emergency Medicine, First Affiliated Hospital, Heilongjiang University of \\ Chinese Medicine, Harbin, China \\ Contributions: (I) Conception and design: R Liu, SM Wang, YL Fu; (II) Administrative support: R Liu, SJ Guo, YL Fu; (III) Provision of study \\ materials or patients: R Liu, SM Wang; (IV) Collection and assembly of data: R Liu, SJ Guo, MM Ma; (V) Data analysis and interpretation: R Liu, \\ SM Wang, SJ Guo, MM Ma; (VI) Manuscript writing: All authors; (VII) Final approval of manuscript: All authors. \\ \#These authors contributed equally to this work. \\ Correspondence to: Yi-Li Fu, MD. School of Life Science and Technology, Harbin Institute of Technology, No. 92 Xi Da Zhi Jie Street, Harbin \\ 150080, China. Email: 3509695830@qq.com.
}

\begin{abstract}
Background: Severe burns, trauma and shock can cause intestinal epithelial barrier dysfunction, which can lead to intestinal endotoxemia and even sepsis and multi-organ dysfunction. Many studies have shown that histone deacetylase inhibitors (HDACIs) can improve cell tolerance to hypoxia and inflammation, thus protecting the functions of important organs in the body, and at the same time, inhibiting the degradation of tight junction (TJ) proteins, protecting the intercellular barrier, and reducing tissue edema and organ damage. However, the mechanism is unclear.
\end{abstract}

Methods: Eighty male Sprague-Dawley rats (weighing 280-300 g) with a 50\% total body surface area fullthickness dermal burn were randomly assigned to 4 groups (20 rats/group): sham control (SC group), scald + normal saline (SN group), scald + 2-methyl-2pentenoic acid (2M2P group), and scald + valproic acid (VPA group). After scalding, we measured the following parameters at various time intervals postburn injury: intestinal mucosal injury score, diamine oxidase (DAO) activity, intestinal protein expression of acetyl histone $\mathrm{H} 3$ at K9 (Ac-H3K9), hypoxia inducible factor $1 \alpha$ (HIF-1 $\alpha$ ), erythropoietin (EPO), zonula occludens-1 (ZO-1), endothelial nitric oxide synthase (eNOS) content, nitric oxide (NO) content, and intestinal mucosal blood flow (IMBF).

Results: Intestinal mucosa showed significant morphologic injury at 4 and 8 hours after scalding that was attenuated by VPA. DAO activity in the VPA group was significantly decreased compared with the other scald groups. At 4 and 8 hours after scalding, VPA enhanced Ac-H3K9 and ZO-1 expression and decreased HIF-1 $\alpha$ and EPO expression in the intestine compared with the other scald groups. At 4 and 8 hours after scalding, eNOS and NO protein content and IMBF in the VPA group were markedly increased compared with the other scald groups.

Conclusions: HDACIs attenuated intestinal mucosal injury in fatally scalded rats. This may have involved VPA enhancing Ac-H3K9 and ZO-1 expression, inhibiting HIF-1 $\alpha$ and EPO expression and inducing eNOS and NO increments.

Keywords: Burns; histone deacetylase inhibitor (HDACI); valproic acid (VPA); hypoxia inducible factor $1 \alpha$ (HIF$1 \alpha)$; zonula occludens-1 (ZO-1)

\footnotetext{
^ ORCID: Rui Liu, 0000-0002-7749-6409; Shu-Ming Wang, 0000-0002-3701-3548; Si-Jia Guo, 0000-0002-3757-5727; Ming-Ming Ma, 0000-0003-1530-7657; Yi-Li Fu, 0000-0002-0968-4766.
} 
Submitted Nov 03, 2021. Accepted for publication Dec 30, 2021.

doi: $10.21037 / \mathrm{atm}-21-5766$

View this article at: https://dx.doi.org/10.21037/atm-21-5766

\section{Introduction}

Traffic accidents, terrorist attacks, and disasters can all result in a large number of patients with burns, trauma, and hemorrhagic shock within a short period of time. Due to limited medical resources and lack of timely treatment available at disaster sites, complications such as infection and shock occur earlier and are more complex, which greatly increases mortality (1-3). Impairment of intestinal epithelial function is a common complication of severe burns, trauma and shock (4). After a severe burn, systemic blood perfusion redistribution, intestinal ischemia and hypoxia, destruction of intestinal mucosal epithelial barrier, translocation of intestinal bacteria, and endotoxin into the blood can result in intestinal endotoxemia and even sepsis and multiple organ dysfunction $(5,6)$.

Several studies have shown that hypoxia inducible factor 1 (HIF-1) plays a key role in hypoxia-induced cell barrier damage $(7,8)$. HIF-1 increases the expression of erythropoietin (EPO), preserves the content of endothelial nitric oxide synthase (eNOS) in endothelial cells, and extracellular signal-regulated kinase (ERK) activates eNOS to produce nitric oxide (NO) (9). Several studies have shown that NO produced by eNOS can regulate gastrointestinal mucosal blood flow and protect the gastrointestinal tract against injury $(10,11)$. It has also been reported that histone deacetylase inhibitors (HDACIs) can protect against burninduced gut barrier dysfunction (12). Both 2-methyl2 pentenoic acid (2M2P) and valproic acid (VPA) are HDACIs; their structures are similar, but the effect of $2 \mathrm{M} 2 \mathrm{P}$ is weaker than that of VPA. Previous studies have found that acetyl histone $\mathrm{H} 3$ at $\mathrm{K} 9$ (Ac-H3 K9) is a reliable index for reflecting the level of histone acetylation (13). Zonula occludens-1 (ZO-1) is a representative protein of the tight junction (TJ) protein family, which is the main factor in the regulation of intestinal epithelial barrier. Reduction in $\mathrm{TJ}$ protein expression and changes in distribution play an important role in the occurrence of functional damage to the intestinal epithelial barrier (14). However, there have been few studies investigating whether VPA-induced NO is involved in increasing intestinal mucosal blood flow (IMBF) and protecting the intestinal mucosal barrier. In addition, it has been reported that HIF-1-induced EPO may provide an adaptive link for the maintenance of barrier function during hypoxia $(7,15,16)$.

VPA has been proven to have protective effects in various hypoxia models $(17,18)$, while $2 \mathrm{M} 2 \mathrm{P}$ has been shown to significantly improve the survival rate of burn shock animals (19). In addition, recent reports show that early use of VPA significantly reduces the degree of brain injury, reduces brain edema, improves neurological dysfunction, and promotes recovery (20). However, the protective effect of VPA on intestinal barrier dysfunction caused by burn has not been confirmed. Therefore, in this study, we aimed to test the hypothesis that, compared with 2M2P, VPA inhibits HIF- $1 \alpha$ and increases eNOS-produced NO, regulating gastrointestinal mucosal blood flow after severe burn, thereby reducing intestinal epithelial barrier dysfunction, histone deacetylation, and ZO-1 expression, thereby reducing intestinal epithelial barrier dysfunction. This study explored the possible mechanism of HDACIs on intestinal function in rats with $50 \%$ total body surface area (TBSA) $\mathrm{III}^{\circ}$ scald injuries in order to provide an experimental basis for the mechanism of HDACIs.

We present the following article in accordance with the ARRIVE reporting checklist (available at https://atm. amegroups.com/article/view/10.21037/atm-21-5766/rc).

\section{Methods}

\section{Animals}

Eighty male Sprague-Dawley rats (specific pathogen-free grade), aged 80-100 days and weighing $280 \pm 20 \mathrm{~g}$ were reared at a constant temperature and humidity in an animal laboratory for 2 weeks to adapt to the environment. Rats were fasted for $12 \mathrm{~h}$ before the experiment, drinking water freely. The animals were provided by Beijing Huafukang Biotechnology Co., Ltd., China, License: SCXK (Beijing) 2019-0008.

\section{Burn procedures}

Rats were skin-prepared and anesthetized with $50 \mathrm{mg} / \mathrm{kg}$ sodium pentobarbital (Sigma-Aldrich, St. Louis, MO, USA). The back and lower limbs of the rats were immersed in boiling water for 15 seconds, and the abdomen was immersed for 8 seconds to induce 50\% TBSA full-thickness $\mathrm{III}^{\circ}$ scald injuries, as described previously (21). The rats in 
the SC group were given sham burns by being immersed in water at $37^{\circ} \mathrm{C}$ for the same period of time. Ethical approval was granted by the Ethics Review Committee of Heilongjiang Provincial Hospital, China (No. 2021-099), in compliance with Chinese national guidelines for the care and use of animals. A protocol was prepared before the study without registration.

\section{Surgical procedures}

At the time intervals of 4 and 8 hours after scalding, the following procedures were performed to measure IMBF. After the rat was anesthetized, an upper-midline laparotomy was performed at 4 and 8 hours after scalding. The $10-\mathrm{cm}$ distal end of the ileocecal area was selected, and IMBF was detected using the infrared probe of a laser Doppler blood flow meter (Perimed AB, Stockholm, Sweden) (22). Perimed software automatically calculated the average blood flow according to the surface area (23). At the end of the experiment, abdominal aortic puncture and exsanguination were performed to achieve euthanasia.

\section{Experimental protocols}

Eighty male Sprague-Dawley rats (weighing 280-300 g) were randomly assigned to 4 groups (20 rats/group): sham control $(\mathrm{SC})$, scald + normal saline $(\mathrm{SN})$, scald $+2 \mathrm{M} 2 \mathrm{P}$ (Sigma-Aldrich), and scald + VPA (Sigma-Aldrich). The rat models with $50 \% \mathrm{TBSA}^{\circ} \mathrm{II}^{\circ}$ scald injuries were prepared using the boiling-water bath method, and the rats in the SC group were given sham burns by being immersed in water at $37^{\circ} \mathrm{C}$. Immediately after scalding, the $2 \mathrm{M} 2 \mathrm{P}$ and VPA groups were given an intraperitoneal injection of $2 \mathrm{M} 2 \mathrm{P}$ or VPA $(300 \mathrm{mg} / \mathrm{kg})$, and the $\mathrm{SC}$ and $\mathrm{SN}$ groups were given normal saline ( $3 \mathrm{~mL} / \mathrm{kg}$, intraperitoneal injection). The scalded rats underwent an abdominal aorta puncture and blood was obtained at 4 and 8 hours after injury, and intestinal tissue was removed from the terminal ileum.

\section{Morphological analysis of intestinal injury}

The terminal ileum tissue sample was frozen at $-80{ }^{\circ} \mathrm{C}$ in liquid nitrogen, embedded in paraffin, cut into $3-\mu \mathrm{m}$ sections for hematoxylin-eosin (HE) staining, and then observed under an optical microscope (Olympus, Tokyo, Japan). Morphological changes were evaluated by 6 pathologists blinded to the experiment. The grading system developed by Søfteland et al. (24) was used to score intestinal injury.

\section{Enzyme-linked immunosorbent assay}

The frozen jejunum mucosa tissues were homogenized and $100 \mathrm{mg}$ of intestinal tissues were placed in a centrifuge tube, incubated on ice for 10 minutes, and then centrifuged at $12,000 \times \mathrm{g}$ for 10 minutes to obtain the supernatant. An enzyme-linked immunosorbent assay (ELISA) was performed to detect diamine oxidase (DAO) activity, eNOS content, and the level of NO in intestinal tissue homogenate according to the kit instructions (Beijing Huasheng Tianchuang Biotechnology Co., Ltd., China).

\section{Western blot assays}

Ac-H3K9, ZO-1, HIF-1 $\alpha$, and EPO proteins were extracted from intestinal tissues. Equal amounts of protein were separated by sodium dodecyl sulfate-polyacrylamide gel electrophoresis and detected by Western blot assays. The proteins were transferred onto polyvinylidene difluoride membranes, and the membranes were blocked with $5 \%$ non-fat dried milk/tris-buffered saline-Tween (TBST) for 1 hour at room temperature. The membranes were then incubated overnight at $4{ }^{\circ} \mathrm{C}$ with anti-Ac-H3 K9 rabbit antibody (diluted 1:400, Abcam, Cambridge, UK); antiHIF-1 $\alpha$ mouse antibody (diluted 1:500, Novus Biologicals, Littleton, CO, USA); anti-EPO mouse antibody (diluted 1:200, R\&D Systems, Minneapolis, MN, USA); and antiZO-1 rabbit antibody (diluted 1:500, Life Technologies, Gaithersburg, MD, USA). Following 3 washes with TBST, the blots were incubated with the secondary antibodies (diluted 1:5,000, ZSGB Biotech, Beijing, China). Finally, immunoblots were detected with a super ECL plus kit (Applygen Technologies Inc., Beijing, China) and visualized after exposure to $\mathrm{X}$-ray film. Densitometer readings were used to quantify the immunoreactive bands. The membranes were reincubated with a mouse monoclonal antibody against glyceraldehyde 3-phosphate dehydrogenase (GAPDH) (diluted 1:5,000, ZSGB Biotech) to control for protein loading. Semiquantitative analysis with image analysis software (Image J) was conducted, and the gray value ratio of the target band and the internal reference band were used to reflect the expression level of the target protein.

\section{Immunofluorescence staining analysis}

After the paraffin sections were routinely deparaffinized, 
hydrated, and rinsed with phosphate-buffered saline (PBS) 3 times, the sections were separated and incubated with $3 \%$ bovine serum albumin (Zhongshan Jinqiao Biotechnology Co., Ltd., Beijing, China) for 30 minutes to block nonspecific binding sites. The sections were then combined with anti-ZO-1 (1:100, Life Technologies) or Ac-H3K9 (1:100, Abcam, Cambridge, UK) overnight at $4{ }^{\circ} \mathrm{C}$. The sections were washed 3 times with PBS and then stained with Alexa Fluor 488 thickened anti-rabbit antibody (1:1,000, Life Technologies) in $1 \%$ bovine serum albumin for 1 hour and then washed 3 times with PBS and antifading solution (Applygen Technologies Inc.). After mounting the slides, images were observed under a fluorescence microscope (BX51-DP71, Olympus). Positive expression of Ac-H3K9 was manifested as a bright green fluorescent signal in the nucleus of the intestinal epithelium, and positive expression of ZO-1 was manifested as a bright green fluorescent signal in the cell membrane between adjacent cells on top of the intestinal epithelium.

\section{Measurement of IMBF}

A laser Doppler flow monitor (PeriFlux 5000 Master, Perimed $\mathrm{AB}$ ) was used to measure IMBF at 4 and 8 hours after scald injury, and a sterile laparotomy was performed along the abdominal alba line. The contact fiber optic probe of the blood flow meter was inserted into the proximal jejunum and fixed along the intestinal canal. The tip of the probe contacted the mucous membrane, measured the flow signal for 30 seconds, and then the instrument automatically calculated the average value. A steady signal of 10 seconds was selected to calculate the mean value. Digital signals were expressed as blood perfusion units (BPU).

\section{Statistical analysis}

Data were analyzed using SPSS version 25.0. Continuous variables are presented as mean \pm standard deviation $(\mathrm{SD})$, the $t$-test was used for intragroup comparison, the $\mathrm{F}$ test for comparison between groups, and the Student-NewmanKeuls test for pairwise comparison between groups. $\mathrm{P}<0.05$ indicated statistically significant difference.

\section{Results}

\section{VPA attenuated scald-induced intestinal mucosal injury}

The functional significance of VPA on intestinal barrier function injury at 4 and 8 hours after scald injury was determined according to the Chiu grading system (25), and the effects of experimental treatment-specific differences on scald-induced villus injuries were assessed. The morphology of the intestinal mucosa of rats in the SC group was intact, while histological evaluations of jejunum mucosal damage in the other groups revealed extensive blunting of the villus tips, the appearance of subepithelial lifting, congestion, and sloughing of the villus tips compared to the SC group. In the $\mathrm{SN}$ group, the intestinal mucosal villi appeared edematous and loosed 4 hours after scalding, and the injury was further aggravated at 8 hours. After $2 \mathrm{M} 2 \mathrm{P}$ treatment, the damage to rat intestinal villi was partially relieved, and after VPA treatment, the damage to rat intestinal villi was significantly reduced (Figure 1). Compared with the SN group, the mean villus injury scores in the $2 \mathrm{M} 2 \mathrm{P}$ and VPA groups decreased significantly at the time intervals of 4 and 8 hours after scalding $(\mathrm{P}<0.05$, respectively, Figure 1). The mean villus injury score in the VPA group at 4 and 8 hours after scalding was significantly lower than that in the $2 \mathrm{M} 2 \mathrm{P}$ group $(\mathrm{P}<0.05$, respectively, Figure 1$)$.

\section{VPA protected DAO activity against scald-induced intestinal mucosa injury}

Intestinal function was evaluated by DAO activity. As shown in Figure 2, compared with the SC group, DAO activity in the other groups was markedly increased at 4 and 8 hours after scald injury ( $\left.{ }^{\mathrm{a}}, \mathrm{P}<0.05\right)$. However, compared with the SN group, DAO activity in the 2M2P and VPA groups was significantly decreased at 4 and 8 hours after scalding ( $\left.{ }^{\mathrm{b}}, \mathrm{P}<0.05\right)$, and DAO activity in the VPA group was significantly lower than that in the $2 \mathrm{M} 2 \mathrm{P}$ group at these time intervals ( ${ }^{\mathrm{c}}, \mathrm{P}<0.05$, Figure 2 ).

\section{VPA increased the expression of Ac- $\mathrm{H} 3 \mathrm{~K} 9$}

Ac-H3K9 reflects well the overall acetylation level of histones (26). Thus, this study examined Ac-H3K9 levels using Western blot and immunofluorescence staining techniques to determine the changes in the overall acetylation levels of histones in intestinal tissues. There was some degree of Ac-H3K9 in all normal rat intestinal mucosal epithelial cells, and immunofluorescence Ac$\mathrm{H} 3 \mathrm{~K} 9$ levels in normal rat intestinal mucosal epithelial cells showed a positive display with a strong fluorescent signal and a continuous state (Figure $3 A, 3 B$ ). However, the fluorescent signal was significantly diminished at 4 and 

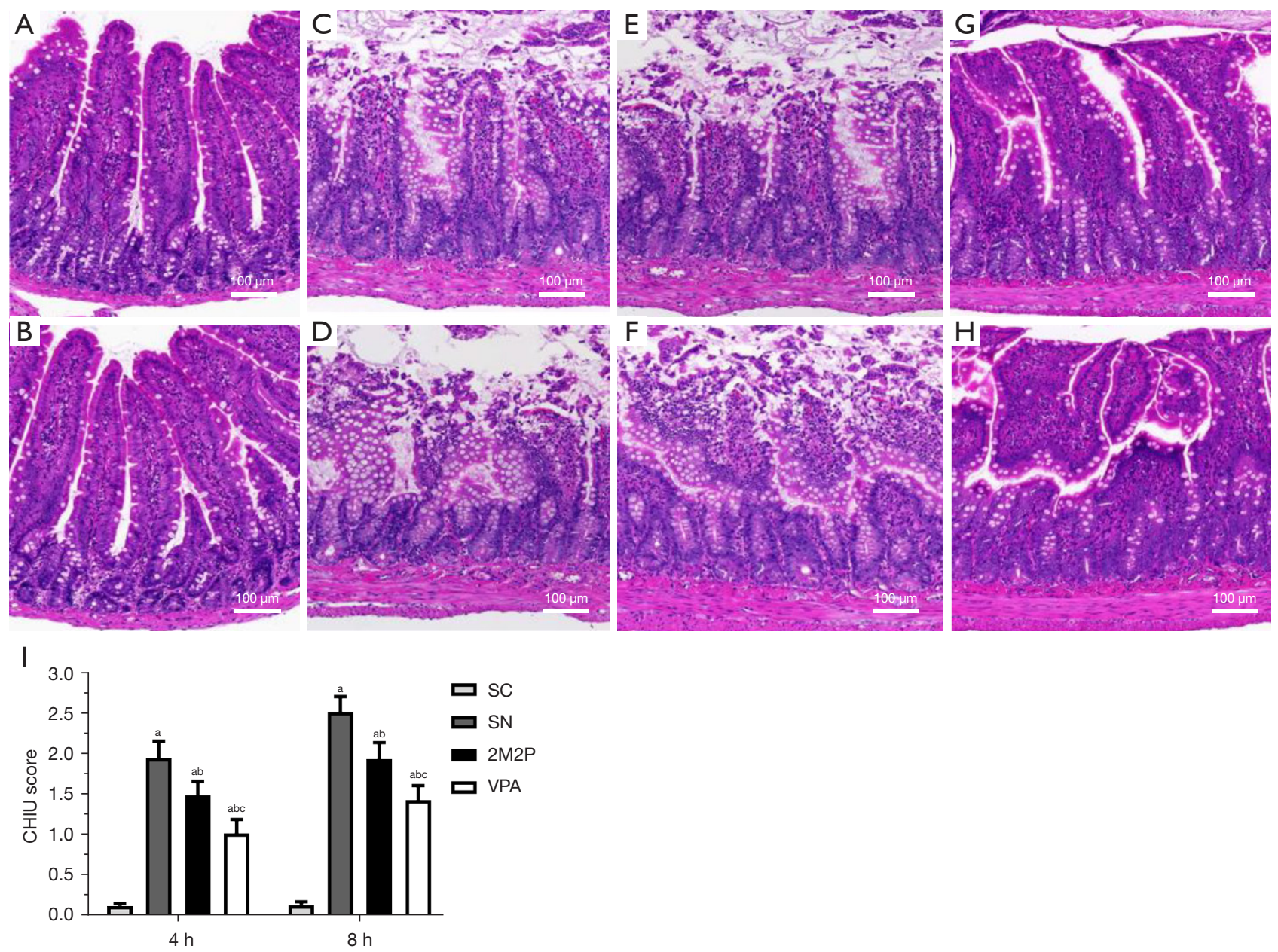

Figure 1 VPA alleviated intestinal mucosal injury caused by scald. The rats in each group were sectioned at 4 and 8 hours post-injury, and the tissue specimens were stained with $\mathrm{HE}$ and then observed under light microscope and selected for ideal field of view for photographing (A-H). (A,B) Representative image of the SC group, showing intestinal mucosal villi were dense, elevated and morphologically intact. (C,D) Representative image of SN group at 4 and 8 hours, respectively; edema and loosening of the intestinal mucosal villi, detachment of a large portion of the intestinal epithelium from the villi, partial detachment of the tip of the villi, gradual progression from the initial enlargement of the subepithelial gap at the tip of the villi (C) and massive separation of the epithelial layer from the lamina propria (D) were observed. (E,F) Representative image of the 2M2P group at 4 and 8 hours, respectively; diminished structural changes in the intestinal mucosal villi can be observed, with no significant elevation on either side of the villi compared with the $\mathrm{SN}$ group, absence of massive lifting down the sides of villi. (G,H) Representative image of the VPA group at 4 and 8 hours, respectively; a significant reduction in damage to the intestinal mucosal villi was observed compared with the $2 \mathrm{M} 2 \mathrm{P}$ group. White bar $=100 \mu \mathrm{m}$. (I) Scoring of intestinal injury using Chiu's grading system. Chiu's scores were significantly higher in rats at both 4 and 8 hours post-burn; 2M2P and VPA treatment reduced Chiu's scores in rats post-injury, while VPA treatment significantly reduced intestinal injury at 4 and 8 hours post-burn. ${ }^{a}, \mathrm{P}<0.05$ vs. SC group; ${ }^{b}, \mathrm{P}<0.05$ vs. SN group; ${ }^{c}$, $\mathrm{P}<0.05$ vs. 2M2P group ( $\mathrm{n}=6-10$ rats at each time interval per group). VPA, valproic acid; TBSA, total body surface area; HE, hematoxylineosin; SC, sham control; SN, scald + normal saline; 2M2P, 2-methyl-2pentenoic acid.

8 hours postburn (Figure 3C,3D), the fluorescence intensity of Ac-H3 K9 in the $2 \mathrm{M} 2 \mathrm{P}$ group was greater than that in the SC group (Figure $3 E, 3 F$ ), and the fluorescence signal intensity of VPA group was better than that of $2 \mathrm{M} 2 \mathrm{P}$ group
(Figure 3G,3H). Western blot analysis of Ac-H3K9 were generally consistent with the trend of immunofluorescence results that VPA intervention significantly increased the overall level of intestinal tissue histone acetylation at 4 and 


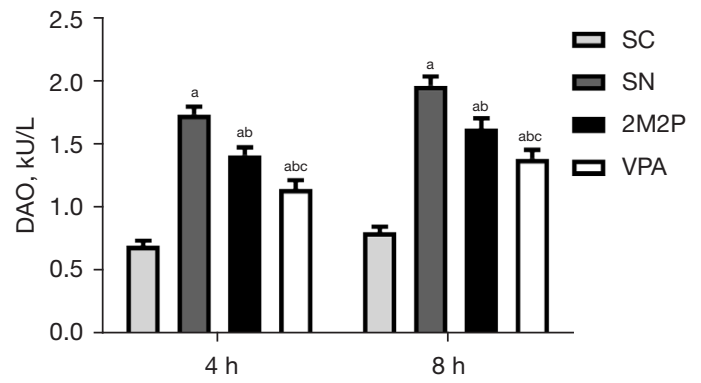

Figure 2 VPA inhibited DAO activity and protected intestinal mucosal injury caused by scald. Application of ELISA for the detection of DAO activity in intestinal tissues. DAO activity was significantly elevated at 4 and 8 hours postburn. 2M2P and VPA treatment significantly reduced the activity of DAO at 4 and 8 hours postburn, while VPA group decreased more significantly. a, $\mathrm{P}<0.05$ vs. SC group; ${ }^{b}, \mathrm{P}<0.05$ vs. $\mathrm{SN}$ group; ${ }^{\mathrm{c}}, \mathrm{P}<0.05$ vs. $2 \mathrm{M} 2 \mathrm{P}$ group ( $\mathrm{n}=6-10$ rats at each time interval per group). DAO, diamine oxidase; SC, sham control; SN, scald + normal saline; $2 \mathrm{M} 2 \mathrm{P}$, 2-methyl-2pentenoic acid; VPA, valproic acid; ELISA, enzymelinked immunosorbent assay.

8 hours postburn (Figure 3I, all $\mathrm{P}<0.05$ ).

\section{VPA protected intestinal barrier function}

To provide evidence that VPA played a protective role in scald-induced intestinal injury, the effect of VPA on the intestinal barrier integrity was examined. As ZO-1 is causally related to intestinal permeability and disturbed by intestinal injury, we tested the hypothesis that intestinal ZO-1 protected by VPA would contribute to the reduction of intestinal injury. As shown in Figure 4, compared with the SC group, ZO-1 protein levels in the scald groups were significantly reduced in intestinal tissue at 4 and 8 hours after scald injury, as demonstrated by Western blot assays. The expression of ZO-1 in the VPA group was significantly higher compared with the other scald groups. Frozen sections for immunofluorescence studies of intestinal epithelial ZO-1 were also prepared. In the SC group, ZO-1 showed a strong and continuous fluorescent signal along the crypt-villus unit, while the fluorescent signal of other groups was not obvious. The ZO-1 protein signal in the $\mathrm{SN}$ group was significantly reduced and even disappeared. The ZO-1 protein signal in the VPA group was lower than that in the SC group, but significantly higher than that in the other groups (Figure 4). These results confirmed that VPA attenuated ZO-1 degradation and intracellular transfer preserved the intestinal barrier integrity.

\section{VPA decreased HIF-1 $\alpha$ and EPO protein expression}

VPA strongly decreased HIF-1 $\alpha$ and EPO in intestinal tissue at 4 and 8 hours after scald injury. Western blot assays demonstrated that each group subjected to burn injury was associated with an increase in HIF- $1 \alpha$ and EPO protein compared with the SC group. The HIF- $1 \alpha$ and EPO protein expression in the $\mathrm{SC}$ group at 4 and 8 hours after scald injury was significantly lower than that in the other groups. The HIF-1 $\alpha$ and EPO protein levels in the VPA group was also lower than that in the other scald groups. The HIF- $1 \alpha$ and EPO protein levels in the 2M2P group were higher than those in the SC and the VPA groups, but lower than those in the $\mathrm{SN}$ group (Figure 5).

\section{VPA enhanced eNOS and NO protein contents}

VPA induced eNOS activity (Figure $6 A$ ) and NO content (Figure 6B). As shown in Figure 6, the contents of eNOS and NO in the VPA group were markedly higher than those in the other scald groups at 4 and 8 hours after scald injury $(\mathrm{P}<0.05)$.

\section{VPA increased the IMBF}

Compared with the SC group, IMBF was significantly decreased in the scald groups at 4 and 8 hours after scald injury. IMBF in the VPA group was significantly higher than that in the other scald groups at 4 and 8 hours after scald injury $(\mathrm{P}<0.05$, Figure 7$)$.

\section{Discussion}

Severe trauma or burn can cause intestinal ischemia/ hypoxia and lead to intestinal barrier dysfunction (27). The integrity of intestinal mucosa is an important index for reflecting intestinal barrier function. In this study, a rat model with $50 \%$ TBSA III $^{\circ}$ scald injury was established, and the changes in intestinal barrier structure and function after severe scalding were determined using the Chiu score. The results showed that the intestinal mucosal villi in rats showed morphological changes such as edema, shortening, widening, and loosening at 4 hours post-scald injury. The degree of intestinal mucosal injury was further aggravated at 8 hours post-scald injury, which was consistent with previous national and international research results $(28,29)$. 

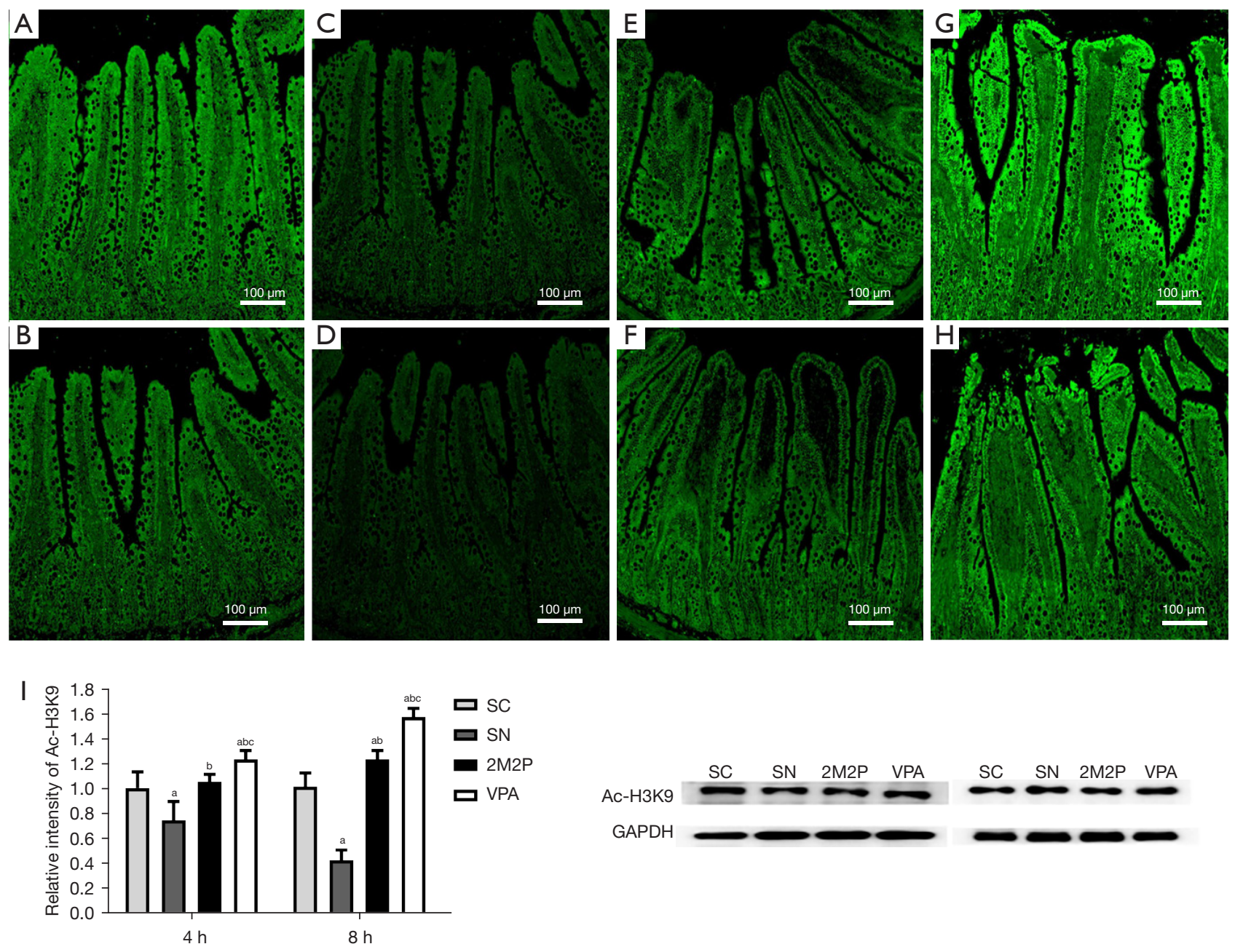

Figure 3 VPA increased the expression of Ac-H3K9. Fluorescence microscopic observation of Ac-H3K9 levels in intestinal tissues of various groups of rats (A-H). (A,B) Representative image of the SC group showing the ubiquitous presence of histone acetylation in normal rat intestinal mucosal epithelial cells, immunofluorescence levels showed a positive display with a strong fluorescent signal and a continuous state. (C,D) Representative image of the SN group at 4 and 8 hours, respectively; significantly weaker immunofluorescence signals and broken junctions in intestinal mucosal epithelial cells can be observed. (E,F) Representative image of the 2M2P group at 4 and 8 hours, respectively; the immunofluorescence signals of Ac-H3K9 in the 2M2P group was greater than that in the SC group. (G,H) Representative image of the VPA group at 4 and 8 hours, respectively; the fluorescence signal of intestinal mucosa was significantly enhanced in the VPA group. White bar $=100 \mu \mathrm{m}$. (I) Detection of intestinal Ac-H3K9 by Western blot. Ac-H3K9 levels were significantly lower in rats at both 4 and 8 hours post-burn; 2M2P and VPA treatment increased Ac-H3K9 levels in rats post-burn, but more significantly in the VPA group. Ac-H3K9 level was calculated by densitometry relative to the housekeeping gene $(G A P D H) .{ }^{a}, \mathrm{P}<0.05$ vs. SC group; ${ }^{b}, \mathrm{P}<0.05$ vs. $\mathrm{SN}$ group; c, $\mathrm{P}<0.05$ vs. $2 \mathrm{M} 2 \mathrm{P}$ group ( $\mathrm{n}=6-10$ rats at each time interval per group). Ac-H3K9, acetyl histone H3 at K9; SC, sham control; SN, scald + normal saline; 2M2P, 2-methyl-2pentenoic acid; VPA, valproic acid; TBSA, total body surface area.

Intestinal epithelial DAO is a highly active intracellular structural enzyme, which is mainly located in intestinal villi. A change in its activity can reflect damage in intestinal mucosal epithelial cells and destruction of intestinal barrier structure. DAO can be directly released into the blood after intestinal tissue damage, and intestinal tissue and blood DAO are negatively correlated. A previous study indicated that the release of DAO from intestinal epithelial cells increased destruction of the intestinal barrier structure (30). The results in this study showed DAO activity in intestinal 

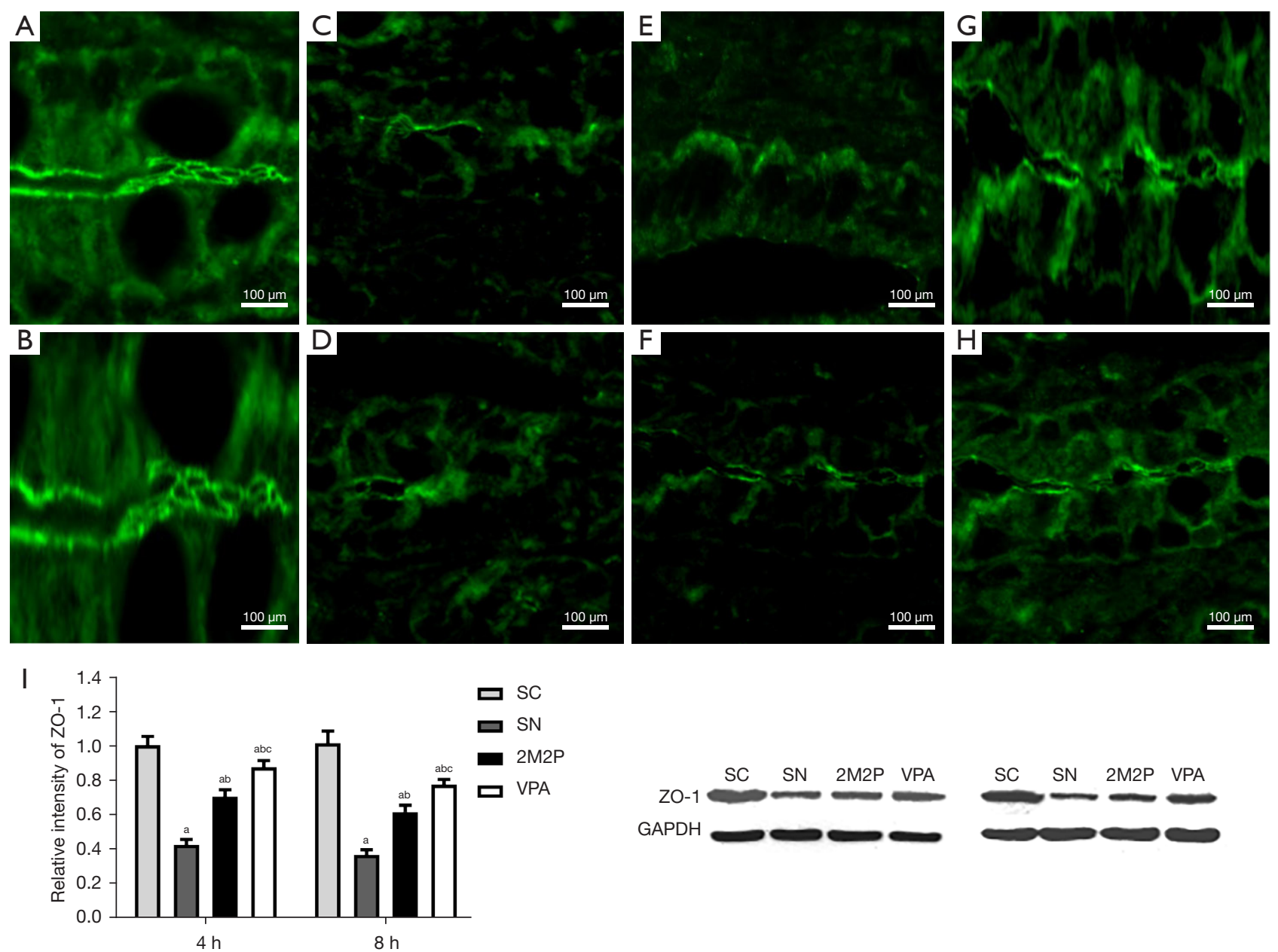

Figure 4 VPA attenuated ZO-1 degradation. The degradation of ZO-1 in intestinal tissues of rats was observed under fluorescence microscope (A-H). (A,B) Representative image of the SC group, normal rats showed positive ZO-1 levels with a strong fluorescent signal and a continuous distribution. (C,D) Representative image of the SN group at 4 and 8 hours, respectively; a significant weakening of the ZO-1 immunofluorescence signal can be observed (C), with a break in the ZO-1 fluorescent band junction (D). (E,F) Representative image of the 2M2P group at 4 and 8 hours, respectively; a slight enhancement of the ZO-1 fluorescence signal can be observed compared with the SN group. (G,H) Representative image of the VPA group at 4 and 8 hours, respectively; significantly enhanced fluorescent signal in the intestinal mucosa can be observed compared with the $2 \mathrm{M} 2 \mathrm{P}$ group. White bar $=100 \mu \mathrm{m}$. (I) Expression of ZO- 1 by Western blot. The expression of ZO-1 was significantly reduced in the scald groups, compared with the SC group. The expression of ZO-1 in the VPA group was significantly higher compared with the other scald groups. The expression of $\mathrm{ZO}-1$ was calculated by densitometry relative to the housekeeping gene $(G A P D H) .^{a}, \mathrm{P}<0.05$ vs. SC group; ${ }^{\mathrm{b}}, \mathrm{P}<0.05$ vs. $\mathrm{SN}$ group; ${ }^{\mathrm{c}}, \mathrm{P}<0.05$ vs. $2 \mathrm{M} 2 \mathrm{P}$ group ( $\mathrm{n}=6-10$ rats at each time interval per group). ZO-1, zonula occludens-1; SC, sham control; SN, scald + normal saline; 2M2P, 2-methyl-2pentenoic acid; VPA, valproic acid.

tissue in the SN group was significantly increased at 4 and 8 hours after scalding, and DAO activity in the VPA group was significantly lower compared with the other scald groups, confirming that VPA reduced DAO activity in intestinal mucosal epithelial cells and protected intestinal barrier function.

With regard to the protective effect of HDACIs on intestinal barrier function, acetylation modification of histone is one of the key mechanisms of gene transcription regulation (31). Under physiological conditions, the acetylation level of histone is jointly regulated by histone acetylases (HATs) and histone deacetylases (HDACs), which display a dynamic balance. However, in the pathological state, the activity of HATs decreases, resulting in disorder 

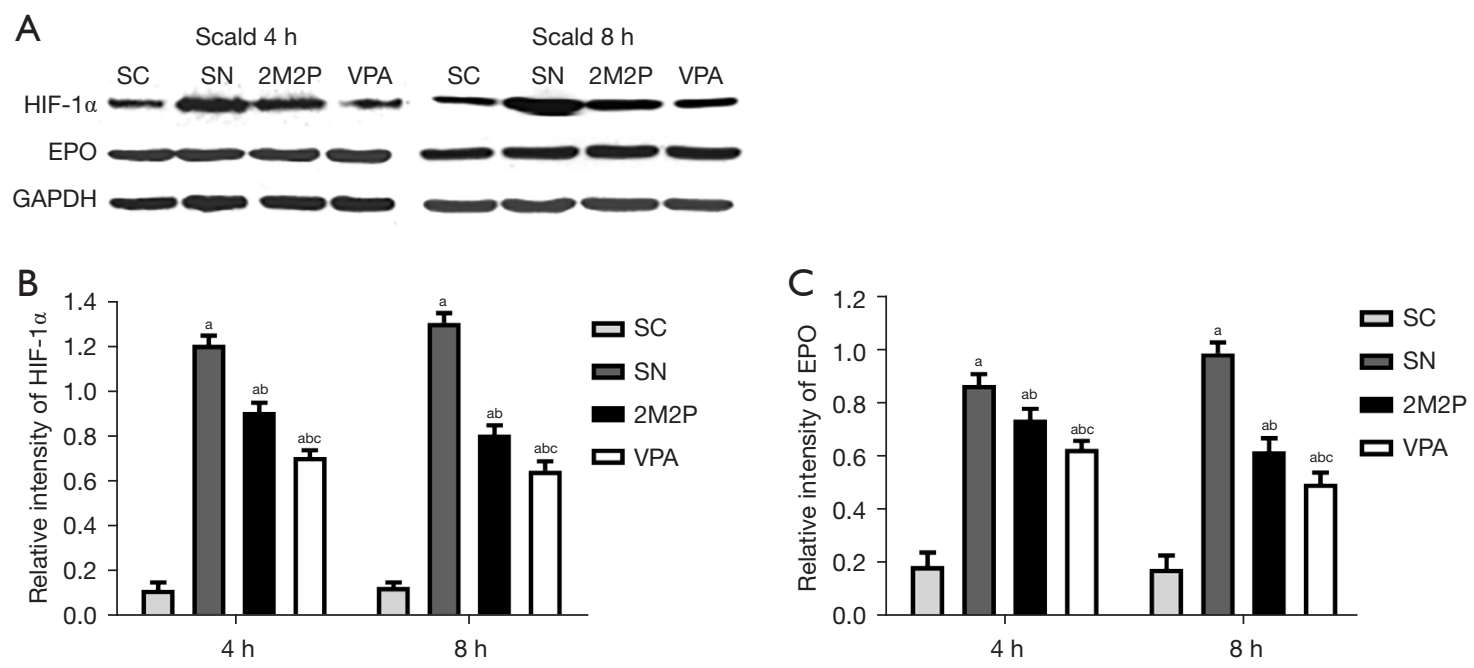

Figure 5 VPA decreased HIF-1 $\alpha$ and EPO protein expression. The expression of HIF-1 $\alpha$ and EPO was determined by Western blot. VPA strongly decreased HIF-1 $\alpha$ in intestinal tissue. HIF-1 $\alpha$ evoked the activity of the EPO protective signaling cascade. The expression of HIF$1 \alpha$ and EPO in intestinal tissues of rats were significantly increased at 4 and 8 hours after scalding; 2M2P and VPA treatment groups reduced the expression of HIF-1 $\alpha$ and EPO in intestinal tissues of rats after scalding, while VPA group reduced them more significantly. HIF-1 $\alpha$ and EPO were calculated by densitometry relative to GAPDH. ${ }^{a}, \mathrm{P}<0.05$ vs. SC group; ${ }^{\mathrm{b}}, \mathrm{P}<0.05$ vs. SN group; ${ }^{\mathrm{c}}, \mathrm{P}<0.05$ vs. $2 \mathrm{M} 2 \mathrm{P}$ group ( $\mathrm{n}=6-10$ rats at each time interval per group). HIF-1 $\alpha$, hypoxia inducible factor $1 \alpha$; SC, sham control; SN, scald + normal saline; $2 \mathrm{M} 2 \mathrm{P}$, 2-methyl-2pentenoic acid; VPA, valproic acid; EPO, erythropoietin.
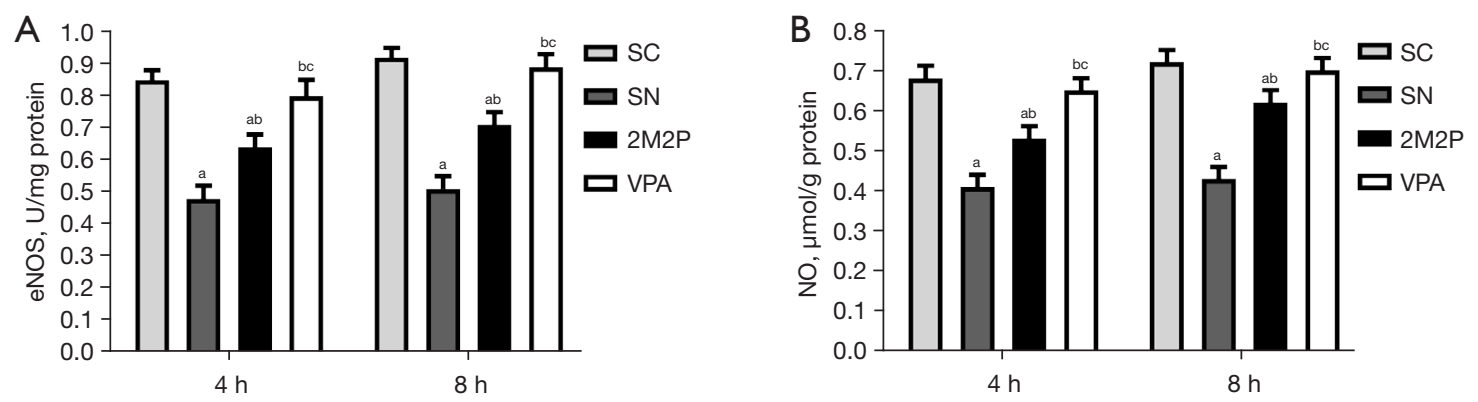

Figure 6 VPA enhanced eNOS (A) and NO (B) protein contents. The contents of NO and eNOS were detected by ELISA at 4 and 8 hours after scalding. The contents of NO and eNOS in the VPA group were markedly increased compared with the other scald groups. a $\mathrm{P}<0.05$ vs. SC group; ${ }^{\mathrm{b}}, \mathrm{P}<0.05$ vs. SN group; ${ }^{\mathrm{c}}, \mathrm{P}<0.05$ vs. $2 \mathrm{M} 2 \mathrm{P}$ group ( $\mathrm{n}=6-10$ rats at each time interval per group). eNOS, endothelial nitric oxide synthase; NO, nitric oxide; SC, sham control; SN, scald + normal saline; 2M2P, 2-methyl-2pentenoic acid; VPA, valproic acid.

of histone acetylation balance and abnormal cell function. Previous studies have shown that HDACIs can improve the tolerance of cells to hypoxia and inflammation and protect the functions of multiple important organs $(17,18)$. The mechanism may be related to acetylation of histone. AcH3K9 can reflect the overall acetylation level of histone (13). Therefore, the level of Ac-H3K9 in intestinal tissue was detected by immunofluorescence and Western blot analysis to assess changes in the overall acetylation level of histone in intestinal tissue. The results showed that the histone acetylation level in the SN group began to decrease at 4 hours after scalding and was more obvious at 8 hours after scalding. VPA rapidly corrected the acetylation imbalance after scald injury in rats, with the change in Ac-H3 K9 observed at 4 hours after scalding.

The TJ is an important structure for maintaining barrier function of the intestinal mucosa. It is located between adjacent cells on the top side of intestinal epithelial cells. It 


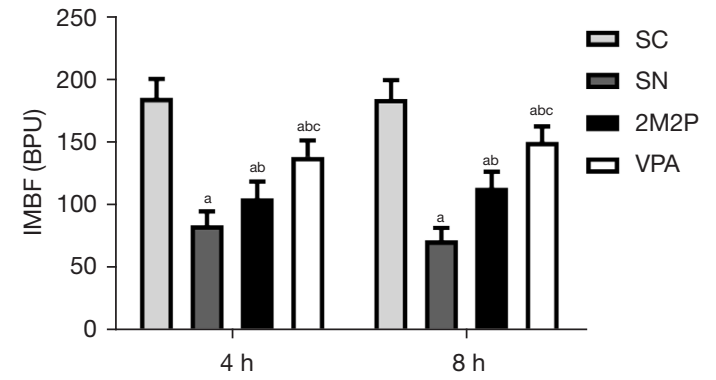

Figure 7 Effects of VPA on IMBF. The effect of VPA on IMBF after burn injury was assessed. IMBF significantly recovered in the VPA group compared with the other scald groups. ${ }^{a}, \mathrm{P}<0.05$ vs. SC group; ${ }^{\text {b }}, \mathrm{P}<0.05$ vs. $\mathrm{SN}$ group; ${ }^{\mathrm{c}}, \mathrm{P}<0.05$ vs. $2 \mathrm{M} 2 \mathrm{P}$ group $(\mathrm{n}=6-10$ rats at each time interval per group). BPU, blood perfusion units; IMBF, intestinal mucosal blood flow; SC, sham control; SN, scald + normal saline; 2M2P, 2-methyl-2pentenoic acid; VPA, valproic acid.

is composed of a variety of TJ proteins, including claudin, occludin, junctional adhesion molecule (JAM), ZO-1, ZO2, and ZO-3 (32). In the past, the TJ was considered to be a fixed static structure, but a previous study showed that the redistribution of $\mathrm{TJ}$ proteins between cells could be caused by hypoxia or inflammation (33). In the present study, immunofluorescence and Western blot analysis were performed to observe the changes in ZO-1 in intestinal mucosal epithelial cells. The results showed that the ZO-1 level began to decrease at 4 hours after scalding and was more obvious at 8 hours after scalding. Additionally, the continuity of some ZO-1 fluorescence bands appeared to be interrupted. This showed that morphological damage to intestinal mucosa occurred within a few hours after severe burns, and the intestinal epithelial TJ protein ZO-1 was degraded and remodeled, resulting in intestinal mucosal barrier dysfunction.

Hypoxia is an important pathophysiological basis for intestinal barrier dysfunction. Studies have confirmed that it involves phosphoinositide-3 kinase (PI3K)/serine-threonine protein kinase (Akt), RAS/Raf-1/mitogen-activated protein kinase kinase 1 (MEK1)/ERK, stress-activated protein kinase (SAPK)/c-Jun N-terminal kinase (JNK), nuclear transcription factor- $\mathrm{\kappa B}(\mathrm{NF}-\kappa \mathrm{B})$, and inducible nitric oxide synthase (iNOS). HIF-1 $\alpha$ is correlated with the signal conditioning pathway (34), but the specific mechanism is not yet fully understood. During hypoxia, the body can recognize hypoxia signals and change the transcription and expression of related genes to adapt to changes in oxygen concentration (35). HIF-1 is an important regulator of oxygen adaptation and can direct the expression of a variety of hypoxia response genes downstream, thereby mediating various pathophysiological changes related to hypoxia (36). HIF-1 is composed of 2 subunits, $\alpha$ and $\beta$, of which $\alpha$ is a functionally active subunit regulated by oxygen concentration. Under hypoxia, the degradation pathway of HIF- $1 \alpha$ is inhibited and forms a dimer with HIF- $1 \beta$, enters the nucleus, binds to the hypoxia response element of its target gene, and then regulates the transcription and translation of related genes (37). In this study, Western blot analysis was used to detect the expression of HIF-1 $\alpha$ in intestinal tissue after burn injury in rats. The results showed that the expression of HIF- $1 \alpha$ in the VPA group was significantly lower than that in the SN group at 4 hours after scalding, and this was more obvious at 8 hours after scalding. The increase in HIF-1 $\alpha$ expression caused by ischemia and hypoxia after scalding may be an important mechanism in the destruction of intestinal epithelial TJ proteins and intestinal epithelial barrier damage after scalding.

$\mathrm{NO}$ is a messenger molecule that plays an important role in regulating cell proliferation, differentiation, and vitality. A previous study showed that when cells were injured due to ischemia and hypoxia, the expression of NO decreased. As a vasodilator, $\mathrm{NO}$ is a protective factor against tissue ischemia and hypoxia after severe burns. It can regulate vascular tone, relax blood vessels, increase vascular permeability, increase blood flow, increase tissue oxygen supply, and further reduce the damage caused by ischemia and hypoxia. In addition, NO can reduce the aggregation of platelets, reduce the adhesion of neutrophils and endothelial cells, remove oxygen free radicals, and maintain vascular permeability (38). In this study, the content of $\mathrm{NO}$ in the $\mathrm{SN}$ group decreased significantly, and the content of NO in the VPA group increased, which was consistent with the above results (38). In normal endothelial cells, eNOS catalyzes the oxidation of $\mathrm{L}$-arginine to produce $\mathrm{NO}$, which participates in regulating vasodilation, platelet aggregation, and leukocyte adhesion, and also protects endothelial cells. When the body's blood oxygen content decreases, eNOS activity changes and NO synthesis decreases or biological activity decreases, leading to the occurrence and development of gastrointestinal diseases (39). The NO synthesized by eNOS can regulate the blood flow of the gastrointestinal tract to reduce the damage caused by gastrointestinal diseases, which improves blood flow and oxygen supply to the ischemic intestinal tissue, protects intestinal mucosal barrier function, and reduces tissue damage (22). During hypoxia, eNOS activity and NO synthesis decrease, and the protective effect on 
endothelial cells is weakened, leading to endothelial cell damage. Our research results showed that compared with the other scald groups, the content of eNOS in the VPA group increased at 4 and 8 hours after scalding, which promoted the synthesis and release of $\mathrm{NO}$, increased blood flow in the intestinal mucosa, and increased the tolerance of intestinal tissue to ischemia and hypoxia, thereby reducing tissue cell damage.

HIF- $1 \alpha$ is an oxygen-sensitive transcription factor that can adapt cells to transient hypoxia (7). A previous study showed that hypoxia can increase the expression of HIF$1 \alpha$. During ischemia and hypoxia, inflammatory mediators and cytokines increase significantly. Under the action of cytokines, eNOS expression decreases significantly (9). This study showed that the level of HIF- $1 \alpha$ significantly increased during tissue ischemia and hypoxia after burn injury, while the level of eNOS decreased significantly, which is consistent with the above data (9). Compared with the other scald groups, the level of HIF-1 $\alpha$ in the VPA group was significantly reduced, while the level of eNOS was significantly increased, which indicated that VPA reversed the increase in HIF- $1 \alpha$ expression caused by hypoxia, upregulated eNOS, and regulated the synthesis and release of NO.

VPA is an HDACI and has a protective effect in a variety of parenchymal cells (17). Previous studies have shown that VPA can inhibit the degradation of a variety of TJ proteins and reduce the permeability and tissue edema of the blood-brain barrier after cerebral ischemia/reperfusion (I/R) injury in rats (40). A previous study has also found that VPA can attenuate cardiac injury and improve survival in a rodent model of lethal burn injury (41). In addition, a variety of HDACIs, including VPA, can inhibit HIF$1 \alpha$ expression (42). HDACI may reduce the inflammatory response and decrease microvascular endothelial cell activation during burns by inhibiting the NF- $\mathrm{BB}$ pathway; HDACI may also reduce endothelial cell damage during severe burns by increasing the expression of the protective protein HSP70, thereby inhibiting the increase in vascular permeability after severe burns, reducing body fluid exudation and improving burn shock. In severe burns, the NF- $\kappa \mathrm{B}$ signaling pathway is activated and reduced HSP70 may be a possible mechanism for the damage to the intestinal epithelial barrier and increased permeability after burns (19). The present study confirmed that the expression of HIF-1 $\alpha$ and EPO in intestinal tissue was significantly increased after scalding. We hypothesized that VPA could inhibit the expression of HIF-1 and EPO and inhibit the degradation of ZO-1. Upregulation of eNOS promotes the synthesis of $\mathrm{NO}$ and increases IMBF, thereby protecting the function of the intestinal mucosal epithelial barrier after severe burns. Therefore, we injected $300 \mathrm{mg} / \mathrm{kg}$ VPA into the abdominal cavity immediately after scalding in rats and observed its effect on intestinal tissue, the level of histone acetylation, and the intestinal mucosal barrier. The results showed that the level of acetylation in the VPA group had already recovered to the level of the SC group at 4 hours after scalding, while the expression of HIF- $1 \alpha$ and EPO was significantly lower than that in the $\mathrm{SN}$ group. The intestinal mucosal Chiu score and the expression of DAO and ZO-1 also improved after VPA treatment, which suggested that VPA may have had a protective effect on the intestinal barrier by inhibiting HIF- $1 \alpha$ and EPO, reducing ZO-1 degradation, activating eNOS, promoting NO synthesis, increasing IMBF, and reducing intestinal barrier damage.

VPA is a common drug in neurology, and as a common drug that has been used clinically for epilepsy treatment, its pharmacological, pharmacokinetic and pharmacological data are relatively clear, as its efficacy for early treatment of severe burn and trauma shock has been confirmed by several studies, but its anti-shock efficacy and dose are still unclear. HDACI, as a novel drug for the early treatment of severe burn and trauma shock, is becoming a new hot spot for unconventional anti-shock treatment research, with promising clinical application and good application prospects, but its domestic and international studies are still relatively few, and its mechanism of action is also rarely reported. In this study, the possible mechanism of the early rescue effect of sodium valproate on severe burn and trauma shock was proposed and preliminarily demonstrated, but it is not very comprehensive and needs further study.

The shortcomings of this paper are that the experimental observation time is still short, and this experiment uses a small animal model of severe burns, and the results obtained are only preliminary, so there is still a need for more animal models for a more realistic in-depth study.

\section{Conclusions}

This study confirmed that severe scalding can cause destruction of the intestinal mucosal TJ protein ZO-1, resulting in intestinal barrier dysfunction and decreased IMBF. After scalding, the level of histone acetylation was significantly decreased, accompanied by an increase in HIF-1 $\alpha$ and EPO expression, which may be an important mechanism of intestinal barrier damage in rats with $50 \%$ 
TBSA III ${ }^{\circ}$ scald injury. VPA restored the level of histone acetylation in intestinal tissue after scalding and inhibited the expression of HIF-1 $\alpha$ and EPO, reduced ZO-1 degradation, activated eNOS, promoted NO synthesis, increased IMBF, and reduced intestinal barrier damage.

\section{Acknowledgments}

Funding: This research was supported by the Special fund of Heilongjiang Provincial and the Chinese Academy of Science and Technology Cooperation Project of China (No. YS20C20).

\section{Footnote}

Reporting Checklist: The authors have completed the ARRIVE reporting checklist. Available at https://atm. amegroups.com/article/view/10.21037/atm-21-5766/rc

Data Sharing Statement: Available at https://atm.amegroups. com/article/view/10.21037/atm-21-5766/dss

Conflicts of Interest: All authors have completed the ICMJE uniform disclosure form (available at https://atm. amegroups.com/article/view/10.21037/atm-21-5766/coif). The authors have no conflicts of interest to declare.

Ethical Statement: The authors are accountable for all aspects of the work in ensuring that questions related to the accuracy or integrity of any part of the work are appropriately investigated and resolved. Ethical approval was granted by the Ethics Review Committee of Heilongjiang Provincial Hospital, China (No. 2021-099), in compliance with Chinese national guidelines for the care and use of animals.

Open Access Statement: This is an Open Access article distributed in accordance with the Creative Commons Attribution-NonCommercial-NoDerivs 4.0 International License (CC BY-NC-ND 4.0), which permits the noncommercial replication and distribution of the article with the strict proviso that no changes or edits are made and the original work is properly cited (including links to both the formal publication through the relevant DOI and the license). See: https://creativecommons.org/licenses/by-nc-nd/4.0/.

\section{References}

1. Liu R, Hu XH, Wang SM, et al. Pyruvate in oral rehydration salt improves hemodynamics, vasopermeability and survival after burns in dogs. Burns 2016;42:797-806.

2. Lin JC, Chen ZH, Chen XD, et al. Circulating sFasL Levels Predict the Severity and Outcome of Burn Injury: A Prospective Observational Study. J Surg Res 2021;265:1-10.

3. Ngaage LM, Borrelli MR, Rose JA, et al. Global Burn Registry Perspective on Head and Neck Burns. Ann Plast Surg 2021;87:33-8.

4. Toppi J, Cleland H, Gabbe B. Severe burns in Australian and New Zealand adults: Epidemiology and burn centre care. Burns 2019;45:1456-61.

5. Hughes A, Almeland SK, Leclerc T, et al.

Recommendations for burns care in mass casualty incidents: WHO Emergency Medical Teams Technical Working Group on Burns (WHO TWGB) 2017-2020. Burns 2021;47:349-70.

6. Tian H, Wang L, Xie W, et al. Epidemiologic and clinical characteristics of severe burn patients: results of a retrospective multicenter study in China, 2011-2015. Burns Trauma 2018;6:14.

7. Ogunshola OO, Al-Ahmad A. HIF-1 at the blood-brain barrier: a mediator of permeability? High Alt Med Biol 2012;13:153-61.

8. Qi H, Wang P, Liu C, et al. Involvement of HIF- $1 \alpha$ in MLCK-dependent endothelial barrier dysfunction in hypoxia. Cell Physiol Biochem 2011;27:251-62.

9. Miller MR, Koch SR, Choi H, et al. Apoptosis signalregulating kinase 1 (ASK1) inhibition reduces endothelial cytokine production without improving permeability after toll-like receptor 4 (TLR4) challenge. Transl Res 2021;235:115-28.

10. da Silva Monteiro CE, Franco ÁX, Sousa JAO, et al. Gastroprotective effects of $\mathrm{N}$-acylarylhydrazone derivatives on ethanol-induced gastric lesions in mice are dependent on the NO/cGMP/KATP pathway. Biochem Pharmacol 2019;169:113629.

11. Spigoni V, Mena P, Cito M, et al. Effects on Nitric Oxide Production of Urolithins, Gut-Derived Ellagitannin Metabolites, in Human Aortic Endothelial Cells. Molecules 2016;21:1009.

12. Luo HM, Du MH, Lin ZL, et al. Valproic acid treatment inhibits hypoxia-inducible factor $1 \alpha$ accumulation and 
protects against burn-induced gut barrier dysfunction in a rodent model. PLoS One 2013;8:e77523.

13. Li Y, Yuan Z, Liu B, et al. Prevention of hypoxia-induced neuronal apoptosis through histone deacetylase inhibition. J Trauma 2008;64:863-70; discussion 870-1.

14. Assimakopoulos SF, Akinosoglou K, de Lastic AL, et al. The Prognostic Value of Endotoxemia and Intestinal Barrier Biomarker ZO-1 in Bacteremic Sepsis. Am J Med Sci 2020;359:100-7.

15. Coimbra-Costa D, Garzón F, Alva N, et al. Intermittent Hypobaric Hypoxic Preconditioning Provides Neuroprotection by Increasing Antioxidant Activity, Erythropoietin Expression and Preventing Apoptosis and Astrogliosis in the Brain of Adult Rats Exposed to Acute Severe Hypoxia. Int J Mol Sci 2021;22:5272.

16. Hirota K. HIF- Prolyl Hydroxylase Inhibitors and Their Implications for Biomedicine: A Comprehensive Review. Biomedicines 2021;9:468.

17. Legrand M, Barraud D, Constant I, et al. Management of severe thermal burns in the acute phase in adults and children. Anaesth Crit Care Pain Med 2020;39:253-67.

18. Lowin J, Winfield T, Price P, et al. Estimating the cost impact of dressing choice in the context of a mass burns casualty event. Ann Burns Fire Disasters 2019;32:222-6.

19. Hu S, Hou JY, Wang HB, et al. The effect of valproic acid in alleviating early death in burn shock. Burns 2012;38:83-9.

20. Wakam GK, Biesterveld BE, Pai MP, et al. Administration of valproic acid in clinically approved dose improves neurologic recovery and decreases brain lesion size in swine subjected to hemorrhagic shock and traumatic brain injury. J Trauma Acute Care Surg 2021;90:346-52.

21. Liu R, Wang SM, Li ZY, et al. Pyruvate in reduced osmolarity oral rehydration salt corrected lactic acidosis in sever scald rats. J Surg Res 2018;226:173-80.

22. Wang ZE, Peng J, Wu D, et al. Effects of intestinal trefoil factor on intestinal mucus barrier in burned mice. Am J Transl Res 2020;12:7187-98.

23. Wang ZE, Wu D, Zheng LW, et al. Effects of glutamine on intestinal mucus barrier after burn injury. Am J Transl Res 2018;10:3833-46.

24. Søfteland JM, Bagge J, Padma AM, et al. Luminal polyethylene glycol solution delays the onset of preservation injury in the human intestine. Am J Transplant 2021;21:2220-30.

25. AlKukhun A, Caturegli G, Munoz-Abraham AS, et al. Use of Fluorescein Isothiocyanate-Inulin as a Marker for Intestinal Ischemic Injury. J Am Coll Surg 2017;224:1066-73.
26. Zhou C, Liu M, Mei X, et al. Histone hypoacetylation contributes to neurotoxicity induced by chronic nickel exposure in vivo and in vitro. Sci Total Environ 2021;783:147014.

27. Muraoka WT, Granados JC, Gomez BI, et al. Burn resuscitation strategy influences the gut microbiota-liver axis in swine. Sci Rep 2020;10:15655.

28. Jiang S, Fan Q, Xu M, et al. Hydrogen-rich saline protects intestinal epithelial tight junction barrier in rats with intestinal ischemia-reperfusion injury by inhibiting endoplasmic reticulum stress-induced apoptosis pathway. J Pediatr Surg 2020;5 5:2811-9.

29. Lysyy T, Finotti M, Maina RM, et al. Human Small Intestine Transplantation: Segmental Susceptibility to Ischemia Using Different Preservation Solutions and Conditions. Transplant Proc 2020;52:2934-40.

30. Zhu H, Xiao X, Shi Y, et al. Inhibition of miRNA29a regulates intestinal barrier function in diarrheapredominant irritable bowel syndrome by upregulating ZO-1 and CLDN1. Exp Ther Med 2020;20:155.

31. Lee JY, Kim H, Jo A, et al. $\alpha$-Synuclein A53T Binds to Transcriptional Adapter 2-Alpha and Blocks Histone H3 Acetylation. Int J Mol Sci 2021;22:5392.

32. Yue D, Wang Z, Yang Y, et al. EZH2 inhibitor GSK343 inhibits sepsis-induced intestinal disorders. Exp Ther Med 2021;21:437.

33. Chen W, Hartman R, Ayer R, et al. Matrix metalloproteinases inhibition provides neuroprotection against hypoxia-ischemia in the developing brain. J Neurochem 2009;111:726-36.

34. Oguzoglu AS, Senol N, Asci H, et al. Pregabalin Protects Brain Tissue from Subarachnoid Hemorrhage by Enhancing HIF-1 $\alpha /$ eNOS Signaling and VEGF Production. World Neurosurg 2021;152:e713-20.

35. Martí-Díaz R, Montenegro MF, Cabezas-Herrera J, et al. Acriflavine, a Potent Inhibitor of HIF-1 $\alpha$, Disturbs Glucose Metabolism and Suppresses ATF4-Protective Pathways in Melanoma under Non-Hypoxic Conditions. Cancers (Basel) 2020;13:102.

36. He H, Chen T, Mo H, et al. Hypoxia-inducible long noncoding RNA NPSR1-AS1 promotes the proliferation and glycolysis of hepatocellular carcinoma cells by regulating the MAPK/ERK pathway. Biochem Biophys Res Commun 2020;533:886-92.

37. Orlando IMC, Lafleur VN, Storti F, et al. Distal and proximal hypoxia response elements cooperate to regulate organ-specific erythropoietin gene expression. Haematologica 2020;105:2774-84. 
38. Lee GH, Park JS, Jin SW, et al. Betulinic Acid Induces eNOS Expression via the AMPK-Dependent KLF2 Signaling Pathway. J Agric Food Chem 2020;68:14523-30.

39. Guan X, Guan X, Lu C, et al. Nebivolol combined with tetrahydrobiopterin affects diastolic function in spontaneously hypertensive rats via the nitric oxide/cyclic guanosine monophosphate signalling pathway. BMC Pharmacol Toxicol 2020;21:84.

40. Bonetti NR, Diaz-Cañestro C, Liberale L, et al. Tumour Necrosis Factor- $\alpha$ Inhibition Improves Stroke Outcome in a Mouse Model of Rheumatoid Arthritis. Sci Rep

Cite this article as: Liu R, Wang SM, Guo SJ, Ma MM, Fu YL. Histone deacetylase inhibitor attenuates intestinal mucosal injury in fatally scalded rats. Ann Transl Med 2022;10(2):54. doi: 10.21037/atm-21-5766
2019;9:2173.

41. Luo H, Hu S, Bai H, et al. The protective effect of valproic acid on myocardium in rats with lethal scald injury and its mechanism. Zhonghua Wei Zhong Bing Ji Jiu Yi Xue 2014;26:563-6.

42. Kim YJ, Park SJ, Kim NR, et al. Effects of Histone Deacetylase Inhibitor (Valproic Acid) on the Expression of Hypoxia-inducible Factor-1 Alpha in Human Retinal Müller Cells. Korean J Ophthalmol 2017;31:80-5.

(English Language Editor: A. Muijlwijk) 